UPR-650-T

\title{
Addendum to Supersymmetric Dyonic Black Holes in Kaluza-Klein Theory
}

\author{
Mirjam Cvetič * and Donam Youm ${ }^{\dagger}$ \\ Physics Department \\ University of Pennsylvania, Philadelphia PA 19104-6396
}

(March 1995)

\begin{abstract}
We complete the study of 4-dimensional (4-d), static, spherically symmetric, supersymmetric black holes (BH's) in Abelian $(4+n)$-d Kaluza-Klein theory, by showing that for such solutions $n$ electric charges $\overrightarrow{\mathcal{Q}} \equiv\left(Q_{1}, \ldots, Q_{n}\right)$ and $n$ magnetic charges $\overrightarrow{\mathcal{P}} \equiv\left(P_{1}, \ldots, P_{n}\right)$ are subject to the constraint $\overrightarrow{\mathcal{P}} \cdot \overrightarrow{\mathcal{Q}}=0$. All such solutions can be obtained by performing the $S O(n)$ rotations, which do not affect the 4-d space-time metric and the volume of the internal space, on the supersymmetric $U(1)_{M} \times U(1)_{E}$ BH's, i.e., supersymmetric BH's with a diagonal internal metric.
\end{abstract}

In Ref. []], 4-dimensional (4-d), static, spherically symmetric, supersymmetric black holes (BH's) in Abelian $(4+n)$-d Kaluza-Klein (KK) theory were obtained with a diagonal internal metric Ansatz. Such BH's correspond to $U(1)_{M} \times U(1)_{E}$ configurations, i.e., they have at most one electric charge $Q$ and one magnetic charge $P$ which necessarily arise from different $U(1)$ groups. Here, we complete the study of 4-d static, spherically symmetric, supersymmetric BH's in Abelian KK theories by addressing the corresponding solutions with a general non-diagonal internal metric Ansatz. We show that $n$ electric charges $\overrightarrow{\mathcal{Q}} \equiv\left(Q_{1}, \ldots, Q_{n}\right)$ and $n$ magnetic charges $\overrightarrow{\mathcal{P}} \equiv\left(P_{1}, \ldots, P_{n}\right)$ of such BH's are subject to the constraint $\overrightarrow{\mathcal{P}} \cdot \overrightarrow{\mathcal{Q}}=0$. All such solutions can be obtained by performing the global $S O(n)$ rotations [2] on the supersymmetric $U(1)_{M} \times U(1)_{E} \mathrm{BH}$ solutions.

Throughout we use the notation specified in Ref. [1].

As discussed in Section 4.1 of Ref. [1], the supersymmetric BH configurations are solutions of the Killing spinor equations, which correspond to the vanishing of supersymmetric

\footnotetext{
*E-mail address: cvetic@cvetic.hep.upenn.edu

$\dagger$ E-mail address: youm@cvetic.hep.upenn.edu
} 
transformations on the dimensionally reduced $(4+n)$-d gravitino, i.e., $\delta \psi_{\mu}^{\mathbf{m}}=\delta \psi_{\tilde{\mu}}^{\mathbf{m}}=0$. Note, $\psi_{\mu}^{\mathbf{m}}$ and $\psi_{\tilde{\mu}}^{\mathbf{m}}\left(\mathbf{m}=1, \cdots, 2^{\left[\frac{n}{2}\right]}, \mu=0, \cdots, 3, \tilde{\mu}=4, \cdots,(n+3)\right)$ are the corresponding 4-d gravitino(s) and dilatino(s), obtained by the dimensional reduction of $(4+n)$-d gravitino(s). (See Eqs. (3.6) and (3.7) of Ref. [1] for the explicit form of the corresponding supersymmetry transformations.)

For spherically symmetric configurations with the 4-d metric Ansatz given by Eq. (4.2) of Ref. [1] and, however, now with a general non-diagonal internal metric Ansatz, one can rewrite the $t$-component $\delta \psi_{t}^{\mathbf{m}}=0$ of the 4 -d gravitino Killing spinor equation, analogous to Eq. (4.9) in Ref. [1], as:

$$
\frac{R}{\sqrt{\lambda}}\left(\partial_{r} \lambda-\frac{1}{\alpha} \lambda \partial_{r} \varphi\right) \gamma^{03} \varepsilon-\sum_{\tilde{a}=4}^{n+3} \tilde{\mathbf{Q}}^{\tilde{a}}\left(\gamma^{35} \otimes \gamma^{\tilde{a}}\right) \varepsilon=0,
$$

where $\tilde{\mathbf{Q}}^{\tilde{a}} \equiv \mathrm{e}^{-\frac{\alpha}{2} \varphi}\left(\Phi^{-1}\right)_{\tilde{\pi}}^{\tilde{a}} Q^{\tilde{\pi}}$. Note, $\alpha=\sqrt{\frac{n+2}{n}}, \Phi_{\tilde{\pi}}^{\tilde{a}}(\tilde{a}, \tilde{\pi}=4, \cdots, n+3)$ is the $n$-bein of the unimodular part of the internal metric, $\varphi$ is the dilaton (the volume of the internal space), and $\lambda$ and $R$ are the $(t, t)$ and $(\theta, \theta)$ components of the 4-d metric $g_{\mu \nu}$, respectively. Here, $Q^{\tilde{\pi}}$ are electric charges obtained by integrating the Maxwell's equation $\partial_{r}\left(\mathrm{e}^{\alpha \varphi} R \lambda \Phi_{\tilde{\pi}}^{\tilde{a}} \Phi_{\tilde{\lambda}}^{\tilde{a}} F^{\tilde{\lambda}} t r\right)=$ 0 .

The $\theta$-component $\delta \psi_{\theta}^{\mathbf{m}}=0$ of the 4-d gravitino Killing spinor equation, analogous to Eq. (4.10), supplemented by the constraint Eq. (5.2) in Ref. [1], which is always satisfied by the Killing spinors of spherically symmetric configurations, assumes the following form:

$$
\left[2 \sqrt{R}-\sqrt{\lambda}\left(\partial_{r} R-\frac{1}{\alpha} R \partial_{r} \varphi\right)\right] \gamma^{13} \varepsilon-\sum_{\tilde{a}=4}^{n+3} \tilde{\mathbf{P}}^{\tilde{a}}\left(\gamma^{25} \otimes \gamma^{\tilde{a}}\right) \varepsilon=0,
$$

where $\tilde{\mathbf{P}}^{\tilde{a}}=\mathrm{e}^{\frac{\alpha}{2} \varphi} \Phi_{\tilde{\pi}}^{\tilde{a}} P^{\tilde{\pi}}$. Here, $P^{\tilde{\pi}}$ are magnetic charges satisfying the Maxwell's equation $\partial_{\theta}\left(\mathrm{e}^{\alpha \varphi} R \lambda \sin \theta \Phi_{\tilde{\pi}}^{\tilde{a}} \Phi_{\tilde{\lambda}}^{\tilde{a}} F^{\tilde{\lambda}} \theta \phi\right)=0$ with $F_{\theta \phi}^{\tilde{\lambda}}=P^{\tilde{\lambda}} \sin \theta$.

Eqs. (1) and (2) can be satisfied if and only if the lower two-component spinors $\varepsilon_{\ell}^{\mathbf{m}}$ and the upper two-component spinors $\varepsilon_{u}^{\mathbf{m}}\left(\left(\varepsilon^{\mathbf{m}}\right)^{T} \equiv\left(\varepsilon_{u}^{\mathbf{m}}, \varepsilon_{\ell}^{\mathbf{m}}\right)\right)$ satisfy the following constraint:

$$
\eta_{Q} \sum_{\tilde{a}=4}^{n+3} \tilde{\mathbf{Q}}^{\prime}{ }^{\tilde{a}}\left(\gamma^{\tilde{a}}\right)_{\mathbf{n}}^{\mathbf{m}} \varepsilon_{\ell}^{\mathbf{n}}=\varepsilon_{u}^{\mathbf{m}}=i \eta_{P} \sum_{\tilde{a}=4}^{n+3} \tilde{\mathbf{P}}^{\prime \tilde{a}}\left(\gamma^{\tilde{a}}\right)_{\mathbf{n}}^{\mathbf{m}} \varepsilon_{\ell}^{\mathbf{n}}, \quad \eta_{Q, P}= \pm 1
$$

where $i \equiv \sqrt{-1}$. Here, $\tilde{\mathbf{Q}}^{\prime} \tilde{a} \equiv \tilde{\mathbf{Q}}^{\tilde{a}} /\left[R \lambda^{-1 / 2}\left(\partial_{r} \lambda-\frac{1}{\alpha} \lambda \partial_{r} \varphi\right)\right]$ and $\tilde{\mathbf{P}}^{\prime} \tilde{a} \equiv \tilde{\mathbf{P}}^{\tilde{a}} /\left[2 \sqrt{R}-\sqrt{\lambda}\left(\partial_{r} R-\right.\right.$ $\left.\left.\frac{1}{\alpha} R \lambda \partial_{r} \varphi\right)\right]$ satisfy the constraint:

$$
\sum_{\tilde{a}=4}^{n+3}\left(\tilde{\mathbf{Q}}^{\prime \tilde{a}}\right)^{2}=\sum_{\tilde{a}=4}^{n+3}\left(\tilde{\mathbf{P}}^{\prime} \tilde{a}\right)^{2}=1
$$

Multiplying the left-most hand side of Eq. (3) by $\sum_{\tilde{b}=4}^{n+3} \tilde{\mathbf{P}}^{\prime} \tilde{b}\left(\gamma^{\tilde{b}}\right)$ and the right-most hand side by $\sum_{\tilde{b}=4}^{n+3} \tilde{\mathbf{Q}}^{\prime \tilde{b}}\left(\gamma^{\tilde{b}}\right)$, and summing the two resultant equations, along with the identity $\left\{\gamma^{\tilde{a}}, \gamma^{\tilde{b}}\right\}=-2 \delta^{\tilde{a} \tilde{b}}$, one has the result:

$$
\sum_{\tilde{a}=4}^{n+3} \tilde{\mathbf{P}}^{\prime} \tilde{\mathbf{Q}}^{\prime \tilde{a}}=0
$$


or equivalently:

$$
\sum_{\tilde{\pi}=4}^{n+3} P^{\tilde{\pi}} Q^{\tilde{\pi}}=\overrightarrow{\mathcal{P}} \cdot \overrightarrow{\mathcal{Q}}=0 .
$$

Therefore, supersymmetric BH's have constrained charge configurations with the electric charge vector $\overrightarrow{\mathcal{Q}}$ and the magnetic charge vector $\overrightarrow{\mathcal{P}}$ orthogonal to one another.

New supersymmetric BH solutions can be generated by performing the global $S O(n)$ rotations on known supersymmetric BH solutions. Namely, the effective 4-d KK Lagrangian density (see Eq. (3.4) of Ref. [四) is invariant under the global $S O(n)$ transformations [2]:

$$
\Phi_{\tilde{a}}^{\tilde{\lambda}} \rightarrow U_{\tilde{\pi}}^{\tilde{\lambda}} \Phi_{\tilde{a}}^{\tilde{\pi}}, \quad A_{\lambda}^{\tilde{\lambda}} \rightarrow U_{\tilde{\pi}}^{\tilde{\lambda}} A_{\lambda}^{\tilde{\pi}}
$$

where $U$ is an $n \times n$ matrix of the $S O(n)$ transformations. Transformations (7) affect the unimodular part of the internal metric and the gauge fields, however, they leave the 4-d space-time metric $g_{\mu \nu}$ and the dilaton $\varphi$ intact. In addition, since the $S O(n)$ transformations are also the symmetry of the Killing spinor equations, the transformed solutions remain supersymmetric, i.e., they also satisfy the Killing spinor equations.

The $S O(n)$ transformations (7) on the $U(1)_{M} \times U(1)_{E}$ supersymmetric BH's generate

solutions with $n$ electric $\overrightarrow{\mathcal{Q}}$ and $n$ magnetic $\overrightarrow{\mathcal{P}}$ charges which are subject to the constraint $\overrightarrow{\mathcal{P}} \cdot \overrightarrow{\mathcal{Q}}=0$ [2]. Therefore, those are all the 4-d static, spherically symmetric, supersymmetric BH's in Abelian KK theories.

\section{ACKNOWLEDGMENTS}

The work is supported by U.S. DOE Grant No. DOE-EY-76-02-3071, and the NATO collaborative research grant CGR 940870. 


\section{REFERENCES}

[1] M. Cvetič and D. Youm, Nucl. Phys. B438, 182 (1995), hep-th \# 9409119.

[2] M. Cvetič and D. Youm, UPR-645-T preprint (1994), hep-th \# 9502099. 